\title{
Spontaneous intracranial dural arteriovenous fistula in the differential diagnosis of pulsatile tinnitus
}

\section{Keywords: Pulsatile tinnitus, Arteriovenous fistula, Case report \\ Introduction}

Tinnitus is defined as the perception of a sound in the absence of a corresponding external source. According to the heart beat synchronization, it can present as Pulsatile Tinnitus (PT) and nonpulsatile Tinnitus (NPT). PT is difficult to diagnose, especially when otoscopy is normal, since the underlying etiology is not always clinically identifiable. Arteriovenous dural fistulas are rare abnormal connections between arteries and veins, corresponding to $10-15 \%$ of cerebral vascular malformations, and may initially manifest as PT. ${ }^{1,2}$

\section{Methods}

The report of this case was submitted and approved by the ethics committee of the Metropolitan University of Santos.

\section{Case presentation}

A 37-year-old man presented with a sudden onset, holocranial headache and left pulsatile tinnitus. The auscultation of the lateral portion of the neck identified a systolic murmur, more intense in the left mastoid region. Otoscopy, fundoscopy, clinical and laboratory examination were normal. History of trauma investigation was negative. Magnetic resonance imaging of the brain, angioresonance and digital subtraction angiography of intracranial vessels confirmed the presence of a dural arteriovenous fistula (Figure 1). The arterial vascular supply was made by cavernous branches of the left internal carotid artery and transverse branches of the left occipital artery, in addition to numerous dural branches from arteries of the contralateral carotid system. The venous drainage was precocious and towards the left transverse and sigmoid sinus into the internal jugular vein and suboccipital plexus in the same topography. The congenital abnormalities in the vascular anatomy of the cerebral arteriovenous transition are the main etiological cause imputed in this case. The patient underwent endovascular treatment with selective arterial embolization with occlusion of the fistulous path and satisfactory results.
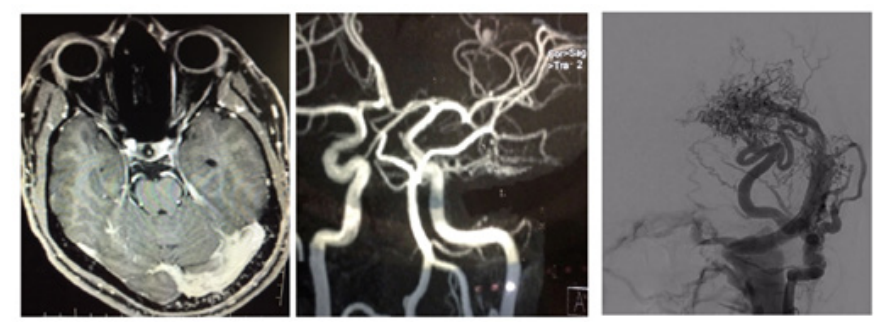

Figure I A) Brain MRI (left), B) Angioresonance of intracranial vessels (middle) and C) Digital subtraction angiography (right) shows a complex dural arteriovenous fistula.

\author{
Volume 8 Issue I - 2018
}

\author{
Joseph Bruno Bidin Brooks,' Mateus Reghin \\ Neto, ${ }^{2}$ Guilherme Lopes da Silveira, ${ }^{3}$ \\ Rodrigo André Oliveira, ${ }^{3}$ Eduardo de \\ Almeida Guimarães Nogueira, ${ }^{4}$ Marcos \\ Vinícius de Queiroz, ${ }^{4}$ Victor Perez Meireles \\ de Souza, ${ }^{4}$ Celso Luis Silva de Oliveira, ${ }^{5}$ \\ Juarez Harding, ${ }^{5}$ Fábio Prosdocimi ${ }^{6}$ \\ 'Department of Neurology, Department of Function and \\ Structure; UNIMES- Universidade Metropolitana de \\ Santos, Brazil \\ ${ }^{2}$ Institute of Neurological Sciences, Hospital Beneficencia \\ Portuguesa de Sao Paulo, Brazil \\ ${ }^{3}$ Clínica Mega Imagem, Brazil \\ ${ }^{4}$ UNIMES- Universidade Metropolitana de Santos, Brazil \\ ${ }^{5}$ Department of Neurology, Irmandade Santa Casa Misericórdia \\ de Santos, Brazil \\ ${ }^{6}$ Department of Function and Structure, UNIMES- Universidade \\ Metropolitana de Santos, Brazil
}

Correspondence: Joseph Bruno Bidin Brooks, Irmandade Santa Casa de Misericórdia de Santos, Avenida Claudio Luiz da Costa 5, Santos, São Paulo, Brazil, I I 75-9, Tel +55- I3-32020600, Email joseph3b@gmail.com

Received: September 12, 2017 | Published: February 15, 2018

\section{Conclusion}

The presentation of this case alerts the possibility of spontaneous intracranial dural arteriovenous fistula in the differential diagnosis of pulsatile tinnitus. Attention to this clinical-radiologic correlation may help physicians make correct diagnoses and appropriate treatment.

\section{Acknowledgements}

None.

\section{Conflicts of interest}

None.

\section{References}

1. Jolly $\mathrm{K}$, Hare $\mathrm{P}$, Irving $\mathrm{R}$, et al. A case of pulsatile tinnitus. BMJ. 2017;356:i6402.

2. Casale M, Sabatino L, Greco F, et al. A reddish pulsatile mass beyond tympanic membrane: think before act. Eur Rev Med Pharmacol Sci. 2016;20(23):4837-4839. 